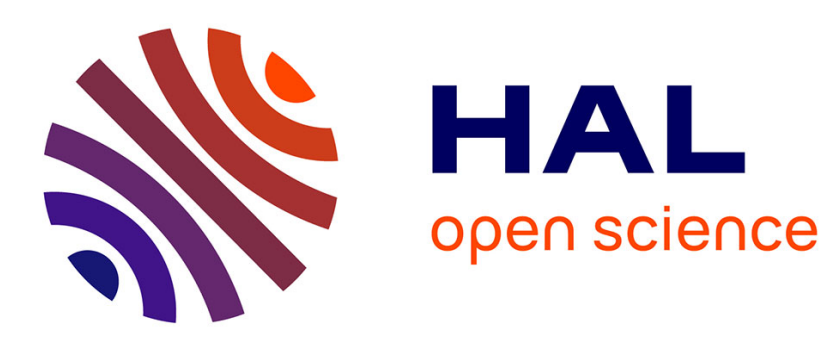

\title{
L'institutionnalisation universitaire de l'enseignement de gestion en France (1965-1975)
}

Fabienne Pavis

\section{To cite this version:}

Fabienne Pavis. L'institutionnalisation universitaire de l'enseignement de gestion en France (1965-1975). Formation Emploi. Revue française de sciences sociales, 2003, p. 51-63, 10.3406/forem.2003.2505 . halshs-02125521

\section{HAL Id: halshs-02125521 https://shs.hal.science/halshs-02125521}

Submitted on 28 Jan 2020

HAL is a multi-disciplinary open access archive for the deposit and dissemination of scientific research documents, whether they are published or not. The documents may come from teaching and research institutions in France or abroad, or from public or private research centers.
L'archive ouverte pluridisciplinaire $\mathbf{H A L}$, est destinée au dépôt et à la diffusion de documents scientifiques de niveau recherche, publiés ou non, émanant des établissements d'enseignement et de recherche français ou étrangers, des laboratoires publics ou privés. 


\section{L'institutionnalisation universitaire de l'enseignement de gestion en} France (1965-1975)

Fabienne Pavis

\section{Citer ce document / Cite this document :}

Pavis Fabienne. L'institutionnalisation universitaire de l'enseignement de gestion en France (1965-1975). In: Formation Emploi. N.83, 2003. pp. 51-63;

doi : 10.3406/forem.2003.2505

http://www.persee.fr/doc/forem_0759-6340_2003_num_83_1_2505

Document généré le 23/03/2016 


\title{
Zusammenfassung
}

Fabienne Pavis, Institutionalisierung universitärer Verwaltungsstudiengänge in Frankreich (1965-1975). Günstige Bedingungen auf internationaler Ebene, Veränderungen in der französischen Hochschullandschaft sowie die Unterstützung und Förderung von Seiten hoher Beamter, Großunternehmer und Hochschullehrer trugen Ende der 60er Jahre zum Auf- und Ausbau der Verwaltungsstudiengänge innerhalb der Wirtschaftswissenschaften bei. Doch es gab auch Gegner, die sich gegen eine Expansion dieser neuen, auf einen nicht „akademischen“ Arbeitsmarkt ausgerichteten Disziplinen aussprachen.

\begin{abstract}
Fabienne Pavis, The institutionalisation of management training at French Universities from 1965 to 1975.

In the late 1960's, the development of business management courses at French universities benefited from favourable international policies, the wind of change blowing at the French universities and the involvement of some high-ranking civil servants, industrial managers and academics, who had been mobilised in advance for these courses. However, there were some who opposed the advent of these new disciplines preparing students for a non-academic labour market.
\end{abstract}

\section{Résumé}

À la fin des années 1960, en France, le développement de l'enseignement universitaire de gestion a bénéficié d'une politique internationale favorable, de la conjoncture de changement de l'université française et de l'engagement de quelques hauts fonctionnaires, grands patrons et universitaires prémobilisés pour cet enseignement. D'autres ont lutté contre l'essor de ces nouvelles disciplines visant un marché du travail non académique. Aujourd'hui, les sciences de gestion constituent une discipline dominante sur le plan quantitatif mais toujours peu visible sur le plan scientifique. 


\title{
L'institutionnalisation universitaire de l'enseignement de gestion en France (1965-1975)

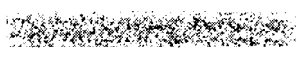

\author{
Savoir bien gérer est un impératif pour tous. Pourtant, l'enseignement de la gestion \\ dans les universités s'est imposé difficilement. Il a du bénéficier de la conjonction \\ d'une politique internationale favorable, des changements de l'université \\ des années 1970, et de l'engagement de quelques hauts fonctionnaires, \\ grands patrons et universitaires.
}

En France, aujourd'hui, les " sciences de gestion » constituent une discipline universitaire qui jouit d'un succès social certain. En effet, elles contribuent à former une part importante des cadres, professions intermédiaires et employés, diffusant ainsi dans différentes sphères sociales les schèmes d'action du management. Les effectifs étudiants en gestion sont près de deux fois supérieurs à ceux d'économie, tandis que le nombre d'universitaires dans les deux disciplines tend à s'équilibrer ( $c f$. encadré 1). Cette inscription universitaire d'une discipline « utile » a contribué aux transformations de l'enseignement supérieur, en particulier sa massification et sa " professionnalisation ». Dans cet article, nous nous proposons d'éclairer les conditions d'institutionnalisation de cette discipline en nous centrant sur la décennie 1965-1975, période durant laquelle une politique active a soutenu cet enseignement tandis que des forces d'opposition l'ont contré, dans un contexte de recomposition universitaire.

Pour saisir le processus qui conduit à l'ancrage des formations en gestion (incluant le marketing, la gestion des ressources humaines, la comptabilité, la finance, etc.) dans les universités au cours des années 1970, il est important de distinguer deux mouvements qui se chevauchent.

D'un côté, au début des années 1960, en France, il existe un ensemble fragmenté d'institutions qui se

Fabienne Pavis est maîre de conférences à l'université de Nantes et associée au Centre de sociologie européenne. Domaines d'investigation : sociologie de l'enseignement supérieur, sociologie des cadres et dirigeants, période : années 1960-1990.

Elle a notamment publié avec M.E. Chessel, Le technocrate, le patron et le professeur. Une histoire de l'enseignement supérieur de gestion, Paris, Belin, 2001, 284 p.

L'article est issu du premier chapitre de la thèse de l'auteur (Pavis, 2003), disponible à la bibliothèque du CNRS de I'IRESCO (Institut de recherche sur les sociétés contemporaines), 59-61, rue Pourchet, 75017 Paris. 
Encadré 1

Les universitaires et étudiants en gestion : données comparatives

\section{Comparaison du nombre et de l'accroissement des universitaires (professeurs et maîtres de conférences) en sciences de gestion et en sciences économiques}

\begin{tabular}{|l|c|c|c|}
\hline & 1994 & 2002 & $\begin{array}{c}\text { Accroissement entre } \\
1994 \text { et } 2002\end{array}$ \\
\hline Sciences de gestion & 716 & 1353 & $+89 \%$ \\
\hline Sciences économiques & 1226 & 1682 & $+37 \%$ \\
\hline
\end{tabular}

Source : Direction des personnels enseignants (DPE), Journée d'étude du Réseau d'étude sur l'enseignement supérieur 7 mars 20031.

Comparaison du nombre et de l'accroissement des étudiants en sciences de gestion, sciences économiques et administration économique et sociale (AES)

\begin{tabular}{|l|c|c|c|}
\hline & 1994 & 2002 & $\begin{array}{c}\text { Accroissement entre } \\
1994 \text { et } 2002\end{array}$ \\
\hline Sciences de gestion & 71914 & 105040 & $+46 \%$ \\
\hline Sciences économiques & 67826 & 55364 & $-18 \%$ \\
\hline AES & 56035 & 53759 & $-4 \%$ \\
\hline
\end{tabular}

Source : note d'information, mars 2003, Direction de la programmation et du développement, MEN.

Remarque : les étudiants en gestion suivent essentiellement des formations professionnelles : la moitié d'entre eux sont inscrits en premier cycle (essentiellement en diplôme universitaire de technologie (DUT) "Gestion des entreprises et des administrations " et "Techniques de commercialisalion "), un liers en second cycle (Institut universitaire professionnalisé (IUP), maitrise de sciences et techniques, maîtrise de sciences de gestion) et $17 \%$ en troisième cycle (essentiellement en diplôme d'études supérieures spécialisées (DESS)|.

Selon la FNEGE (Fondation nationale pour l'enseignement de la gestion des entreprises), qui retient une définition extensive des formations universitaires en gestion (incluant les formations en gestion des IEP (instituts d'études politiques), AES (Administration économique et sociall, etc.), la proportion d'étudiants occupant des postes de cadres trois ans après le diplôme sur la période 1996-1999 se répartit comme suit : parmi les diplômés de premiers cycles, près de la moitié sont cadres ou occupent une profession intermédiaire; parmi les seconds cycles, environ la moitié deviennent cadres; et cette proportion est de deux tiers pour les troisièmes cycles (FNEGE, 2000).

préoccupent de formation en gestion : les écoles de commerce, créées à partir du XIX siècle, qui ont quasiment le monopole des formations initiales, les Instituts d'administration des entreprises (IAE) qui, à partir de 1955, proposent une formation complémentaire d'un an en gestion au sein des facultés de droit et sciences économiques, et de nombreux instituts offrant ce que l'on nomme alors le "perfectionnement à l'administration des entreprises ». Initiatives privées, publiques ou consulaires, associant des ressources patronales et scolaires, plus ou moins influencées par le management des États-Unis (de l'OST - Organisation scientifique du travail - des années 1930 à la politique de productivité des années 1950), ces institutions diverses diffusent des techniques et savoirs depuis les sciences humaines jusqu'aux mathématiques appliquées, en passant par le contrôle de gestion et les techniques de commercialisation. Ce domaine foisonnant mais marginal n'est pas perçu comme un tout, hormis par les organismes planificateurs. Il est investi par des acteurs hétérogènes pour qui il s'agit d'une occupation secondaire : consultants, économistes, juristes, comptables, psychosociologues, ingénieurs, hauts fonctionnaires, patrons... Ces formations existantes et ces acteurs prémobilisés (en particulier les universitaires 
des $I \Lambda E$ constituent) l'une des bases du développement de la gestion dans les universités à la fin des années 1960.

De l'autre côté, les formations en gestion bénéficient de transformations qui touchent l'ensemble de l'enseignement supérieur et notamment les universités. À la fin des années 1960, la massification relative des effectifs étudiants et la contestation du fonctionnement universitaire rendent envisageable, voire souhaitable pour certains acteurs en position de "réformateurs technocrates ", le développement de formations à visée pratique de type non juridique. Une politique offensive de promotion de la gestion est mise en place. Elle est symbolisée par la création, en 1968, de la FNEGE (Fondation nationale pour l'enseignement de la gestion des entreprises), la construction d'une université (Paris IX-Dauphine) consacrée à la gestion et l'économie appliquée, mais aussi par la mise en place de nombreux diplômes nationaux préparant à des fonctions de gestion. La gestion se renforce à la fois au sein du système universitaire renouvelé, où elle devient une discipline " comme une autre », et dans le système des grandes Écoles où elle acquiert une position plus favorable, en particulier par rapport aux écoles d'ingénieurs.

Pour comprendre l'institutionnalisation universitaire des formations en gestion en France, en intégrant ce double mouvement, il est nécessaire de s'intéresser aux enjeux économiques qui y sont associés et à la promotion au niveau international du " modèle » des Business Schools nord-américaines. Nous examinerons ensuite comment la recomposition universitaire issue des mouvements de 1968 va paradoxalement faciliter l'entrée de la gestion à l'université ; l'exemple du Centre universitaire de Dauphine permettra de préciser quels étaient les acteurs individuels et collectifs favorables ou hostiles à l'enseignement universitaire de gestion. Enfin, nous verrons dans quelle mesure les écoles de commerce se rapprocheront des filières universitaires.

\section{L'ATTRAIT DU « MODÈLE » DES BUSINESS SCHOOLS}

L'essor des formations en gestion est à mettre en relation avec la politique de productivité. Alors que le Commissariat général à la productivité et l'Agence européenne de productivité (AEP) ont favorisé le développement de formations en gestion visant des cadres et ingénieurs en poste (Boltanski, 1980 ; Kipping, Nioche, 1997), les organismes qui leur font suite, respectivement le Commissariat général au Plan (qui remplace le Commissariat à la productivité en 1959) et l'OCDE (Organisation de coopération et de développement économiques) créée en 1961 (qui absorbe l'Organisation européenne de coopération économique dont l'AEP faisait partie intégrante), se préoccupent de la croissance des formations initiales' ${ }^{1}$.
"La formation scientifique des managers $[\ldots]$ est

perçue comme un

facteur de développement socioéconomique et une source de stabilité politique dans un contexte de Guerre

froide. »
L'examen de deux rapports internationaux diffusés en France permet de préciser l'argumentaire utilisé pour transformer l'enseignement supérieur de gestion? Les rapports Platt et Grégoire se situent dans une visée prospective caractéristique de l'expertise du début des années 1960, alliant des représentants du « patronat éclairé » (diplômé, international...) à des universitaires et hauts fonctionnaires « modernistes ». L'intérêt des auteurs pour l'éducation des cadres et dirigeants prend sens dans le credo international dominant de l'éducation adaptée à l'économie, étayé par un nouveau savoir, l'économie de l'éducation, que l'OCDE a contribué à diffuser parmi les cercles décisionnels (Papadopoulos, 1994 ; Halsey, Karabel, 1977). Dans les rapports Platt et Grégoire, les pays européens sont comparés avec «les deux puissances mondiales " sous l'angle de la proportion de jeunes poursuivant des études au-delà de dix-huit ans et

\footnotetext{
' La recherche de M.-E. Chessel sur la genèse de la FNEGE montre bien cette inflexion (Chessel, Pavis, 2001).

2 L'un, publié en 1963 sous l'égide de LOCDE. Problèmes et perspectives de la formation à la gestion des entreprises en turope. dirigé par le patron anglais d'une grande entreprise internationale. la Shell, J. W. Platt, lautre de I'UNESC O (1964), Les sciences sociales dans l'enseignement supérieur: Administration des entreprises, rédigé par Roger Grégoire, Conseiller d'État qui a dirigé l'AEP de 1954 à 1961 et qui a auparavant été un réformateur de l'administration française en qualité de directeur de la Fonction publique après guerre.
} 
apparaissent comme "sous-développés $»^{3}$. Les systèmes éducatifs européens sont appelés à se réformer et à prendre davantage en considération l'enseignement d'administration des entreprises en formation initiale et permanente. La formation scientifique des managers a des vertus à la fois économiques - adapter les pratiques des cadres et dirigeants à un nouveau contexte (le marché européen, la concentration des entreprises... ) - et démocratiques former des dirigeants de métier, en opposition implicite au seul héritage. Elle est perçue comme un facteur de développement socio-économique et au-delà, une source de stabilité politique dans un contexte de Guerre froide.

Pour ces auteurs, les Européens doivent nécessairement s'intéresser au mode d'enseignement de l'administration des entreprises aux États-Unis, pays où il est le plus répandu et où une " scientifisation des savoirs " est en cours. Au milieu des années 1960, plus de 500 établissements y forment au management, principalement au sein de Colleges à un niveau de premier cycle (quatre années d'études qui, après la High School dont on sort à dix-sept ans, permettent d'obtenir le diplôme de Bachelor in Business administration) et marginalement à un niveau de second et troisième cycles avec le Master et le doctorat $(P h D)$ spécialisés en Business administration. Au début des années 1960, les fondations Ford et Carnegie, actrices des politiques éducatives américaines, impulsent un renouveau de cet enseignement jugé de piètre qualité. Les management sciences, avec en particulier l'application des méthodes mathématiques et statistiques aux problèmes de gestion et d'organisation, doivent supplanter l'empirisme ambiant dans les Business Schools. Les fondations préconisent une élévation du niveau de formation avec, en particulier, le développement des $P h D$ en Business administration et la généralisation du statut d'enseignant-chercheur, aux dépens des enseignants praticiens (Cochoy, 1999).

Grâce à ces réformes en cours, les experts européens sont convaincus d'une possible conciliation entre la tradition universitaire européenne (allemande), basée sur la rigueur intellectuelle et une forme d'universa-

\footnotetext{
3 Professeur de sociologie à Lausanne, P. Jaccard, qui partage le rationalisme optimisme des années 1960, évoque les enjeux de ce qu il nomme «la stérile guerre froide des statistiques éducatives et professionnelles " : les systèmes éducatifs étant profondément différents, les comparaisons sont biaisées (Jaccard, 1962).
}

lisme, et la tradition des États-Unis affirmant clairement des fins utilitaires ${ }^{4}$. À leurs yeux, alors que le premier atout du système nord-américain de management education est, malgré le faible niveau des études de premier cycle, une insertion universitaire qui va de soi, le second atout réside dans l'existence de quelques formations en administration des entreprises de haute qualité, au sein des Graduate Business Schools, écoles autonomes mais intégrées dans les universités. Trois orientations sont distinguées - celle de Harvard, de Carnegie Tech. et de Chicago - , le modèle est donc pluriel.

Dès lc milicu des annécs 1960, de nombreuses actions s'engagent dans plusieurs pays d'Europe pour y développer les formations en gestion dans l'enseignement supérieur (Whitley, Thomas, Marceau, 1981 ; Engwall, Gunnarsson, 1994). Le "modèle » des Business Schools a joué un rôle dans l'élaboration de la réforme de cet enseignement; cependant, suivant les configurations nationales et les rapports de force internes, il a été approprié différemment, de même que l'action des fondations américaines en Europe (Gemelli, 1998). Dans le contexte français, ce " modèle " est pris en compte par certaines élites modernisatrices. La création des Instituts universitaires de technologie (IUT), à partir de 1966, s'inspire des Colleges nord-américains avec notamment les sections «Administration et gestion des entreprises et des collectivités » et «Techniques de commercialisation » (Bernard, 1970). De même, la FNEGE, conçue dans une commission du Plan en 1966 et mise en place deux ans plus tard, a pour première mission de former un corps enseignant permanent, spécialisé en gestion, chargé de revaloriser et de renouveler les filières existantes. Si la gestion universitaire s'appuie sur des forces politiques nationales et internationales qui font la promotion d'un enseignement jugé nécessaire au développement économique, elle bénéficie également d'un mouvement qui la dépasse : le mouvement de 1968 contribue à légitimer de nouvelles disciplines, dont la " gestion des entreprises », bientôt rebaptisée " sciences de gestion ».

\footnotetext{
4 Le cas de la psychologie est exemplaire, avec notamment le " marché » des tests d'intelligence (armée, écoles...) qui contribue à légitimer la discipline (Paicheler, 1992).
} 


\section{LA GESTION DANS LA RECOMPOSITION UNIVERSITAIRE DE 1968}

En France, avant 1968, les enseignements universitaires de gestion sont quantitativement et symboliquement marginaux. Ils sont transmis dans la quinzaine d'instituts d'administration des entreprises dirigés par des économistes et juristes non nécessairement spécialistes de ces questions, ainsi que dans les sections « tertiaires » des récents IUT alors peu légitimes ; dans ces filières, les professeurs proviennent essentiellement de l'enseignement technique (un CAPET - Certificat d'aptitude professionnelle à l'enseignement technique - et une agrégation forment aux techniques de gestion). Quant à la réforme du cursus de sciences économiques (19541960), elle a très peu bénéficié aux enseignements de gestion. Dans le diplôme d'études supérieures rénové, la comptabilité d'entreprise (couplée avec la comptabilité nationale) est optionnelle, alors que dans la nouvelle licence en quatre ans, aucun enseignement de ce type n'est dispensé (Flouzat, 1963). Pour les professeurs d'économie alors minoritaires', l'enjeu consiste à s'autonomiser du droit et à développer une science formalisée éclairant les décideurs au niveau macro-économique et non à diffuser des techniques de gestion à de futurs cadres. Mais à partir de 1968-1969, les bouleversements du système universitaire français vont favoriser l'essor de cette discipline à vocation professionnelle dans les cycles longs.

De nombreux débats ont lieu, au milieu des années 1960, autour de la massification et de la démocratisation de l'enseignement secondaire et supérieur, le degré de spécialisation des filières. la sélection à l'entrée pour contrer le taux d'échec important aux examens, les débouchés des filières traditionnelles, la place des nouvelles disciplines, les objectifs de la recherche, l'organisation des carrières, la marge de manœuvre des facultés (Passeron, 1986; Prost, 1997 ; Boltanski, 1980). Les manifestations organi-

\footnotetext{
Sn 1964. les facultés de droit et de sciences économiques comptent un quart d'économistes $(\mathrm{N}=138)$ et trois-quarts de juristes $(\mathrm{N}$ $=400$ ). Marco, 2001. Une trentaine dentre cux s"intéressent à la gestion des entreprises.
}

sées en 1967 et 1968 contre le Plan Fouchet-Aigrain" mais aussi contre la guerre du Vietnam ou encore contre les conditions de vie des étudiants aboutissent à une grève générale au milieu du mois de mai 1968 . C'est dans un contexte d'urgence que le nouveau ministre de l'Éducation nationale, Edgar Faure, négocie une réforme universitaire tenant compte des différents acteurs en présence. Distinguer comme le fait J.-C. Passeron trois pôles, les réformateurs, les conservateurs, les contestataires - dont la qualification est potentiellement une disqualification - permet de clarifier les rapports de force et leur évolution : alors qu'au milieu des années 1960, les " technocrates réformateurs $\gg$ qui tentent d'imposer leurs vues sont contestés aussi bien par les syndicats étudiants et enseignants de gauche ( contestataires ») que par les professeurs « conservateurs » (qui souhaitent le statu quo) au printemps 1968, ce sont ces professeurs qui sont mis au pilori par les « contestataires » de gauche et par les " réformateurs ». Enfin, à partir de l'été 1968, « conservateurs » et « réformateurs » s'opposent aux fractions étudiantes et enseignantes qui refusent le principe des examens puis de la reconstruction des universités (Passeron, 1986).

La loi d'orientation sur l'enseignement supérieur, construite autour de trois notions floues aux vertus de cohésion - l'autonomie, la participation et la pluridisciplinarité -, est votée à l'unanimité en octobre 1968. Elle fait éclater les anciennes facultés par la constitution d'unités de base dites « unités d'enseignement et de recherche » (UER) regroupées ensuite au sein d'universités conçues comme des établissements à vocation pluridisciplinaire disposant d'organes de décision et de délibération propres. Une redistribution des pouvoirs administratifs est prévue

\footnotetext{
"Cette réforme dite Fouchet-Aigrain, du nom du ministre en charge et du directeur des Fnseignements supéricurs. est mise en chantier en 1963, sur des principes de spécialisation scientifique et professionnelle des filières, de révision des cursus et des programmes, de contrôle du nombre des flux étudiants par la sélection à l'entrée des facultés (Passeron, 1986, p. 374).
} 


\section{« La création du}

\section{Centre universitaire de}

Dauphine marque une

politique claire en

faveur de l'essor de

l'enseignement de

gestion » au profit des enseignants ne possédant pas le statut de professeur mais aussi des étudiants et du personnel non enseignant.

À partir de mai 1969 , les UER appelées à fonder les premières universités sont listées et validées par le ministère. Dans les grandes villes, plusieurs universités sont créées et une nouvelle organisation se met progressivement en place, souvent de façon conflictuelle dans un climat jugé plus ou moins anarchique (Minot, 1983). La gestion est affectée par ces réformes : une partie des IAE et des IUT sont constitués en unités d'enseignement et de recherche autonomes, tandis que certaines UER de sciences économiques et de gestion, distinctes des unités juridiques, sont établies.

Parallèlement, les économistes et juristes investis dans l'enseignement de la gestion vont bénéficier d'une organisation propre de leur carrière. Depuis 1945, le Comité consultatif des universités, qui gère les carrières des universitaires, est organisé uniquement en cinq divisions (droit et sciences économiques, lettres et sciences humaines, sciences, médecine, pharmacie). Dans le groupe juridique scindé en quatre sous-divisions (droit privé, droit public et sciences politiques, droit romain et histoire du droit, économie politique), la gestion n'apparaît pas. À la faveur d'une reconnaissance de la diversité des disciplines, le nombre des sections est élargi à quarante-sept en mai 1969. Sont créées une section " gestion des entreprises » ainsi que des sections de linguistique, sciences de l'éducation, psychiatrie, génie civil, mécanique, etc.

La même année, le concours d'agrégation du supérieur qui organise l'accès au statut de professeur dans les disciplines juridiques, est partiellement réformé. La reconnaissance de la gestion se confirme dans le nouveau règlement du concours : la section d'économic s'intitule désormais « sciences économiques et de gestion » et l'une des leçons porte au choix sur la politique économique ou la gestion des entreprises. Une agrégation du supérieur en sciences de gestion est instituée par un texte de mai 1975, offrant la possibilité aux spécialistes de ce domaine d'obtenir des postes de professeur, statut indispensable pour peser dans l'organisation universitaire.

Les sciences de gestion bénéficient de la recomposition de l'après-68 et ainsi se normalisent et s'autonomisent comme discipline. Mais cette existence institutionnelle reste fragile tant qu'il n'existe pas de corps enseignant spécialisé en gestion et que ceux qui s'investissent dans ce domaine (notamment en enseignant dans les IAE et départements tertiaires d'IUT) demeurent minoritaires dans les conseils de facultés et autres commissions qui font le quotidien des décisions universitaires. Le Centre expérimental de Dauphine, créé en 1968, (parallèlement à ceux de Vincennes et de Luminy à Marseille) va permettre d'accélérer le développement universitairc de la gestion. Sa création soulèvera des oppositions.

\section{POUR OU CONTRE LE CENTRE UNIVERSITAIRE DE DAUPHINE}

La création du Centre universitaire de Dauphine marque une politique claire en faveur de l'essor de l'enseignement de gestion ; cependant, des acteurs qui n'y sont pas nécessairement favorables interviennent de façon directe ou indirecte dans sa conception et sa mise en œuvre : les professeurs qui dominent les facultés de droit et de sciences économiques et entendent conserver leur pouvoir, et les étudiants et enseignants qui contestent une discipline jugée " au service de l'industrie $»$.

Durant l'été 1968, Edgar Faure charge une commission d'experts de concevoir, pour la rentrée, un centre universitaire dans les anciens locaux de l'OTAN (Organisation du traité de l'Atlantique Nord) situés Porte Dauphine. Ce centre doit permettre « le délestage " des facultés de droit et des sciences économiques; de même, il vise la mise en place d'un enseignement interdisciplinaire à caractère principalement économique, orienté vers la formation aux tâches de gestion et d'administration des collectivités et entreprises publiques ou privées (activités dites du " tertiaire $»)^{7}$. Cette commission est composée de deux responsables d'entreprises publiques « modernisateurs »-F. Bloch-Lainé, inspecteur des finances, directeur de la Caisse des dépôts et consignations et

Archives privées P. Tabatoni, "Rapport de la commission OTAN ». 
M. Boiteux nouveau directeur général d'Électricité de France (après en avoir été directeur des Études économiques à la direction générale) - et de deux jeunes agrégés de sciences économiques alors conseillers au cabinet du ministre, A. Bienaymé, professeur à Dijon et A. Cotta, professeur à Caen et à HEC (Hautes études commerciales). Elle est présidée par le doyen honoraire de la faculté de droit et de sciences économiques, G. Vedel, alors président du syndicat autonome.

Le court rapport de cette commission OTAN préconise la création d'un premier, second et troisième cycles et de dix départements affirmant une orientation professionnelle (en particulier économie de l'entreprise, comptabilité et finances, informatique, commerce et marketing, droit social et relations humaines). Dans ce projet, le contrôle doit être continu; de même, les cours, organisés essentiellement en petits groupes, doivent ainsi allier expérimentations pédagogiques souhaitées par le ministère et contraintes liées au bâtiment doté d'une centaine de petites salles ${ }^{\times}$.

Pour mettre en place ce nouveau centre, dont l'ouverture est prévue en fin d'année, E. Faure choisit un chargé de mission "réformateur ", crédible auprès des professeurs « conservateurs », P. Tabatoni. Agrégé d'économie politique (1950), spécialiste d'économie financière, fondateur de l'IAE d'Aix-en-provence (1956), proche de Gaston Berger (ancien directeur de l'Enseignement supérieur, promoteur d'un rapprochement unversités-entreprises), promu à la faculté de droit et de sciences économiques de Paris à trenteneuf ans (1962), co-auteur du Rapport Platt, il est choisi certes pour sa connaissance du domaine de l'administration des entreprises mais également parce que, en qualité de professeur de la faculté de droit et de sciences économiques de Paris, il est en position de négocier la création de cette nouvelle faculté parisienne et d'empêcher toute velléité de contrôle par les doyens en place".

Les promoteurs du centre de Dauphine ont pu utiliser des outils juridiques pour contrer les forces d'opposition internes à l'université. En effet, quelques

\footnotetext{
"Cf. B. (Girod de l'Ain (1968). "Le nouveau centre universitaire Dauphine ouvrira avant la fin de novembre ". Le .Monde. 30 octobre.

"Entretien avec Pierre Tabatoni du 22 septembre 1998 et Tabatoni, 1996.
}

semaines avant la suppression des facultés (12 novembre 1968), le décret du 24 octobre 1968 créé le Centre universitaire Dauphine "ayant statut de faculté ». Un arrêté du 27 décembrc 1968 fixc l'organisation des « études de gestion et d'économie appliquée $»$ de ce centre expérimental. Une prérogative permet à l'établissement (contrairement aux autres) de déterminer «les programmes, la nature et la durée des enseignements, les modalités du contrôle des connaissances des candidats et les conditions d'assiduité des étudiants ». Dans ce cadre, P. Tabatoni impulse sa conception des « sciences des organisations " sur l'un des " modèles " nord-américains. celui de Carnegie Tech ${ }^{10}$. Dès 1968 à Dauphine, plusieurs diplômes spécialisés en gestion sont créés à titre expérimental, puis sont institués au niveau national : la maitrise d'informatique appliquée à la gestion des entreprises (1970), la maîtrise de sciences de gestion (1971), la maîtrise de sciences et techniques comptables et financières (1974) et le doctorat de troisième cycle et d'État en gestion (1974). Le centre universitaire, qui devient l'université Paris IXDauphine au printemps 1970 (lorsque les 131 UER parisiennes sont rassemblées en 13 universités), se construit sur des bases nouvelles.

Dans un climat où l'entreprise et l'État sont contestés, reste à trouver des étudiants et des enseignants pour faire fonctionner cette institution expérimentale. Le

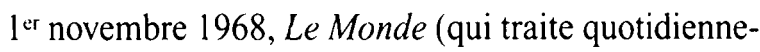
ment des universités) se fait l'écho de la conférence de presse donnée par M. Boiteux, A. Cotta et P. Tabatoni : cette " nouvelle université tertiaire " a pour objectif de "donner aux étudiants une formation de base solide, de leur apprendre à appliquer des connaissances à des problèmes concrets et de les préparer au changement $"^{11}$. Ils doivent en effet contrer le discours des militants d'extrême-gauche, notamment ceux du PAN (Panthéon-Assas-Nanterre) qui affirment sur des tracts affichés à l'entrée du centre que les étudiants y recevront "une pseudoculture qui fera [d'eux] des cadres moyens ».

Le recrutement des universitaires est également problématique. Une quarantaine de professeurs,

\footnotetext{
11. Sont créés en particulier un département de mathématiques appliquées à la décision puis une UER de sciences des organisations. Arrêtés du 13 janvier et du 31 mai 1969.

"Girod de l'Ain (1968). "Le nouveau centre Dauphine préparera à la "gestion des organisations" ". Le Monde. lor novembre.
} 
maîtres de conférences et maîtres-assistants et une centaine d'assistants sont engagés en trois semaines ${ }^{12}$. Si une partie d'entre eux sont particulièrement motivés pour travailler dans ce cadre expérimental, ce n'est pas le cas de tous. Des assistants " gauchistes » étaient présents à Dauphine, qu'ils aient «noyauté " de leur propre chef cet établissement ou qu'ils y aient été envoyés par les professeurs de la faculté de droit parisienne pour saborder le projet concurrent (ou tout simplement se débarrasser d'assistants jugés encombrants). Selon P. Tabatoni, ils étaient une soixantaine mobilisés pour "empêcher que ça fonctionne » et il dit avoir négocié avec les leaders la partition du Centre : aux assistants les premiers cycles et aux professeurs les seconds et troisièmes cycles. En octobre 1969, le licenciement de six assistants dont cinq militants du SNESUP (Syndicat national de l'enseignement supérieur) fait éclater, hors du centre pilote, les conflits qui s'y déroulent (Le Figaro et Le Monde les mentionnent) $)^{13}$.

Le Centre Dauphine, impulsé par les « réformateurs » ne se constitue pas ex nihilo. Les décisions politiques (textes juridiques émis, locaux attribués), permettent en grande partie de contourner les pouvoirs universitaires en place et ainsi de mettre en œuvre une nouvelle filière de diplômes professionnels en quelques mois ; en revanche, pour fonctionner, celleci nécessite un accord implicite des doyens et des enseignants motivés, ce qui ne va pas de soi.

Ainsi, à la fin des années 1970, le bilan de la gestion à l'université demeure mitigé. Certes, avec les sections " tertiaires » d'IUT, l'université Paris IXDauphine, les UER de sciences économiques et de gestion qui préparent aux diplômes nationaux de gestion, l'agrégation du supérieur spécifique et les IAE qui perdurent, la gestion devient une véritable filière dans le système universitaire renouvelé ( $c f$. encadré 2 ). Et la FNEGE a contribué à ce renforcement par son action de formation de spécialistes de gestion en Amérique du Nord et à Bruxelles (Chessel,

\footnotetext{
${ }^{12}$ Sclon Le Monde, on compte parmi les enseignants titulaires une majorité d'économistes (21), des mathématiciens-statisticiens (8), des juristes (6), des anglicistes (4) et des psychosociologues (4). B. Girod de l'Ain, ibid. Sur les conditions de recrutement dans une université expérimentale occupant une position opposée à Dauphine (Vincennes), cf. Soulié, 1998.

${ }^{13}$ Cf. Bulletin du SNESUP, n“ 185, octobre 1969 et $\mathrm{n}^{\circ} 186$, novembre 1969.
}

Pavis, 2001). Néanmoins, le développement est relatif : les sciences de gestion ne disposent pas de DEUG (diplôme d'études universitaires générales), le nombre de postes d'enseignants-chercheurs en gestion demeure faible (dans une période de restriction des moyens), l'université de Paris IX-Dauphine, principale pourvoyeuse de docteurs en sciences de gestion jusqu'au début des années 1980, est encore peu prestigieuse, etc. Ce renouveau universitaire, quelles que soient ses faiblesses, affecte le monopole des écoles de commerce.

\section{LES ÉCOLES DE COMMERCE : DE L'ENSEIGNEMENT TECHNIQUE À L'ENSEIGNEMENT SUPÉRIEUR}

Les bouleversements universitaires ont modifié le fonctionnement des écoles de commerce, même si le système des grandes Écoles, avec l'année préparatoire et le concours, n'est pas remis en cause durant cette période de réforme. Entre le milieu des années 1960 et le milieu des années 1970, celles-ci évoluent de façon différente suivant leurs instances de rattachement et leur notoriété. Dans ce secteur moins centralisé, il faut distinguer les transformations des écoles parisiennes, occupant le haut de la hiérarchie, des transformations du réseau des écoles supérieures de commerce qui dépendent des chambres de commerce et d'industrie locales. Ces évolutions concernent en particulier l'organisation des institutions, le contenu des programmes de formation et des concours d'entrée, les locaux et les caractéristiques des enseignants.

Dans le courant des années 1960, à HEC, aboutissent des réformes engagées à partir de 1957, tandis que son transfert à Jouy-en-Josas est réalisé entre 1964 et 1966. Une réforme de fond est envisagée afin de créer un Master in Business Administration (MBA). Il s'agit d'ouvrir l'école à des diplômés et de jeunes cadres d'horizons divers, en tenant compte davantage des motivations pour les affaires que d'un concours jugé déconnecté des préoccupations entrepreneuriales. L'objectif est de faire acquérir des compétences de gestionnaires grâce à des enseignants spécialisés qui utiliseront la méthode des cas. À ce projet très influencé par la Harvard Business School, soutenu par le directeur G. Lhérault et quelques jeunes enseignants formés eux-mêmes à Harvard et 


\section{Encadré 2}

\section{L'essor de l'enseignement de gestion à l'université et hors université}

Entre le milieu des années 1950 ef le milieu des années 1970, de nombreuses certifications, commissions, institutions sont créées, relatives à l'enseignement et la recherche en gestion (nous n'avons pas reporté les créations des nouvelles écoles supérieures de commerce : les ESC de Poitiers, Amiens, Brest, Nice, Pau, créées entre 1961 et 1969). Le terme " d'administration des entreprises " qui apparaît dans les universités en 1955, sera repris dans le sigle des ESC mais de fait peu utilisé. Le terme de " gestion des entreprises ", relativement neutre, est présent sur toute la période. L'expression * techniques de gestion " est peu utilisée dans les certifications. L'intitulé * sciences de gestion "apparaît en 1968 dans le cadre des diplômes du nouveau Centre universitaire Dauphine.

\begin{tabular}{|c|c|c|}
\hline 1950 & Dans les universités & $\begin{array}{l}\text { Hors des universités } \\
51 \text { : Certifical d'aptitude professionnelle à l'enseigne- } \\
\text { ment technique (CAPET) * Gestion des entreprises et } \\
\text { des collectivités " } \\
53 \text { : Stage d'étude ce la gestion des entreprises } \\
\text { (STEGE) }\end{array}$ \\
\hline 1960 & $\begin{array}{l}55 \text { : Certifical d'aptitude à l'administration des entre- } \\
\text { prises; Institut d'administration des entreprises (IAE) } \\
63 \text { : Doctorat sciences économiques mention * Adminis- } \\
\text { tration des entreprises * }\end{array}$ & $\begin{array}{l}55 \text { : Institut d'études supérieures des techniques d'orga- } \\
\text { nisation (IESTO) } \\
56 \text { : Institut de contrôle de gestion (ICG) } \\
58 \text { : Institut européen d'administration des affaires } \\
\text { (INSEAD) } \\
59 \text { : Centre lyonnais d'étude de la gestion des entre- } \\
\text { prises (CLEGE) } \\
62 \text { : Agrégation des lechniques économiques de } \\
\text { gestion (TEG) }\end{array}$ \\
\hline 1965 & 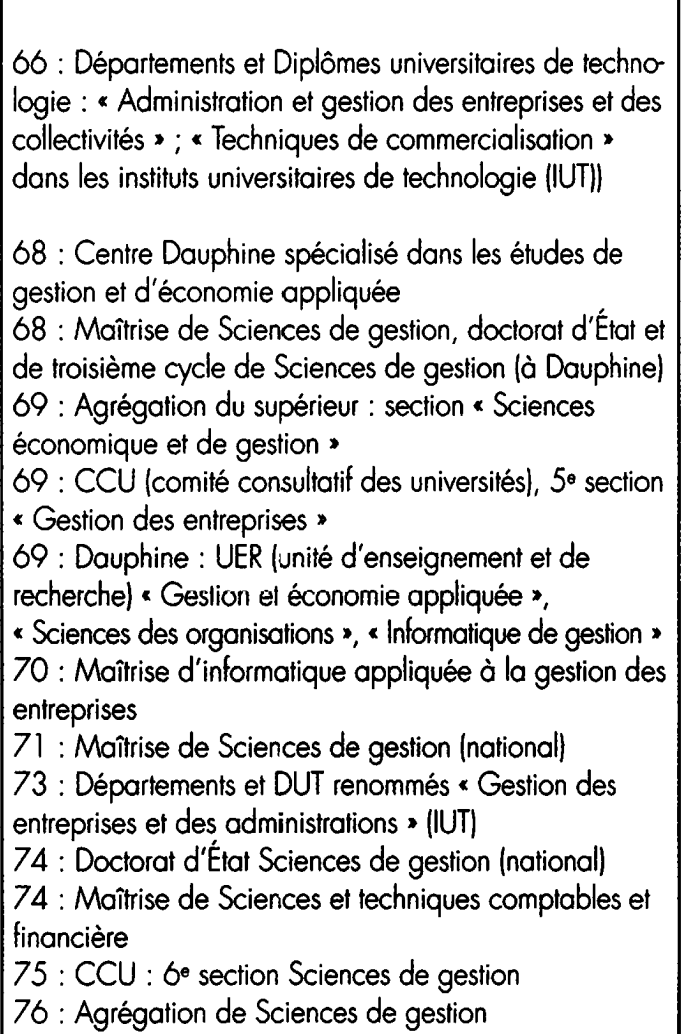 & $\begin{array}{l}65 \text { : Baccalauréats techniques: } G 1 \text {, *Techniques } \\
\text { administratives *; G2, "Techniques quantitatives de } \\
\text { gestion "; G3, "Techniques commerciales " } \\
65: \text { les Écoles supérieures de commerce (ESC) sont } \\
\text { renommées * Écoles supérieures de commerce et } \\
\text { d'administration des entreprises * (ESCAE) } \\
68: \text { Fondation nationale pour l'enseignement de la } \\
\text { gestion des entreprises (FNEGE) } \\
69: \text { numéro de la revue Avenirs: "La gestion des } \\
\text { entreprises. Formation et fonctions ". } \\
69: \text { Institut supérieur des affaires (ISA), au sein de } \\
\text { HEC (École des hautes études commerciales) }\end{array}$ \\
\hline
\end{tabular}


Stanford, s'opposent finalement l'association des anciens élèves puis bientôt la direction de la Chambre de commerce et d'industrie de Paris (CCIP).

En 1969, l'Institut supérieur des affaires (ISA) qui naît de ce projet, ne remplace pas mais complète l'école des HEC. Celle-ci, qui a difficilement acquis son statut de " grande École » ne peut sans doute pas se permettre de risquer tout son capital symbolique accumulé, dans une initiative d'allure révolutionnaire. Elle perdure tout en renouvelant son concours et son programme. Un premier noyau de professeurs permanents, formés aux États-Unis (en partie avec l'aide de la FNEGE), est constitué. En 1970, une structure administrative, le CESA (Centre d'enseignement supérieur des affaires) englobe l'ensemble des instituts : l'École des HEC, l'Institut supérieur des affaires et l'Institut de formation continue, et deviendra bientôt le Groupe HEC ${ }^{14}$.

Les autres écoles sont directement affectées par ces changements. L'ESCP (École supérieure de commerce de Paris), dont la direction consulaire a envisagé la fusion avec HEC, s'autonomise face au réseau des écoles supérieures de commerce et crée ses propres concours et examens de sortie ${ }^{15}$. Alors que les jeunes filles ont désormais le droit de se présenter aux concours d'HEC et de l'ESCP, HEC « Jeunes filles " disparaît. Une nouvelle école de la CCIP, l'École des affaires de Paris (EAP), est créée en 1973 pour promouvoir " l'international » désormais orienté vers l'Europe et les États-Unis et non plus vers les anciennes colonies. Toujours du côté des écoles parisiennes, mais cette fois dans l'enseignement privé, l'ESSEC (École supérieure des sciences économiques et commerciales) entre également dans une ère de changement ; ce qui se manifeste en particulier par le renouvellement des enseignants et le déménagement dans la ville nouvelle de CergyPontoise en 1973. LINSEAD (Institut européen d'administration des affaires), pourtant forgé sur le modèle nord-américain (de Harvard), se transforme au début des années 1970 : un corps enseignant permanent est créé et le $M B A$ s'impose. Ces écoles bénéficient

\footnotetext{
it Sur le projet de l'ISA, (ff. le témoignage de Roland Reitter (in de Saint-Martin, Dinu Gheorghiu, 1997, pp. 72-74) et Nouschi, 1988.

is Archives de la CCIP, $176 \mathrm{~W} 16$, réunion du 20/11/1968 de la commission permanente des écoles supćricures de commerce.
}

également des séjours de formation organisés par la FNEGE.

Aiguillonnées par le mouvement de réforme des écoles parisiennes mais aussi par l'intérêt soudain de l'université pour ces formations, les autres écoles consulaires qui sont à la fois plus directement liées aux Pouvoirs publics et au patronat local, se rénovent à partir du milieu des années 1960. En 1965, elles s'inspirent des IAE en se nommant écoles supérieures d'administration des entreprises et non plus écoles supérieures de commerce; administrativement, elles dépendent désormais de l'enseignement supérieur et non de l'enseignement technique. Le concours national régissant les ESCAE est modifié le baccalauréat est exigé de tous les candidats, le passage en classe préparatoire devient indispensable, le niveau de mathématiques est sensiblement élevé et quelques années plus tard,

«La formation continue est intégrée

[...] dans les

départements

universitaires où elle

permet d'accroitre les

ressources propres

mais surtout le crédit

entrepreneurial » le programme de formation intègre les " techniques modernes de gestion ». Les ESC, auparavant dénigrées (en particulier dans les rapports internationaux sur la formation des dirigeants d'entreprises) car on leur reproche un niveau scolaire trop faible et une dépendance vis-à-vis d'un patronat jugé archaïque, tendent à s'imposer dans le paysage de l'enseignement supérieur. De nouvelles écoles s'établissent dans leur sillon : entre 1967 et 1974, sept écoles dépendant de chambres de commerce et onze écoles privées se créent ${ }^{16}$. L'acquisition d'un capital scolaire spécifique s'impose (Bourdieu, 1989).

Dans les écoles d'ingénieurs, en particulier celles qui avaient déjà manifesté leur intérêt pour la gestion, de nouvelles initiatives émergent. L'école des Mines de Paris propose une démarche de recherche qui aboutit à la création du Centre de gestion scientifique (CGS) en 1967, tandis que l'École polytechnique fonde le Centre de recherche en gestion (CRG) en 1972. Au

\footnotetext{
in Cf. Anmuaire national des écoles de commerce et des formations. supérieures de gestion, L'Étudiant, 1992.
} 
Conservatoire national des arts et métiers, la chaire d'organisation scientifique du travail se dédouble en 1970 : apparaissent les chaires d'organisation du travail et de l'entreprise et de théorie et système d'organisation. Un département d'économie et gestion est constitué parallèlement à un département de sciences de l'homme au travail dans l'entreprise.

L'autre vecteur de transformation important concerne l'institutionnalisation de la formation continue. La législation de 1971 permet aux institutions de formation initiale de se lancer de façon plus offensive sur le marché lucratif des formations de cadres et dirigeants. Elles entrent ainsi en concurrence avec les instituts privés qui, eux aussi, tentent d'élargir leur champ d'action. Cette intervention étatique sur la demande de formation contribue à la restructuration de l'offre désormais pensée comme telle.

En effet, à partir du début des années 1970, le domaine de la formation en gestion est fortement institutionnalisé et ses différentes fractions interagissent beaucoup plus qu'auparavant. La création des doctorats de gestion à l'université est suivie par la mise en place d'un programme doctoral à HEC (1974), de troisièmes cycles alliant l'ESSEC et l'IAE d'Aix (1974) d'une part et l'école supérieure de commerce de Lyon et les universités de Lyon II et Lyon III (1979) d'autre part. Ces accords permettent aux écoles d'acquérir une légitimité savante qui devient progressivement indispensable dans cet univers. La formation continue est intégrée dans les écoles de commerce constituées en groupes et dans les départements universitaires, où elle permet d'accroitre les ressources propres mais surtout le crédit entrepreneurial. Enfin, les accords internationaux (échanges d'étudiants, doubles diplômes, etc.) sont développés dans les ćcoles de commerce, puis dans les universités, pour bénéficier d'une légitimité internationale progressivement valorisée dans un monde économique marqué par le développement du marché européen et des multinationales (Lazuech, 1999).

Une politique de promotion de la gestion comme discipline de l'enseignement supérieur émerge au milieu des années 1960. Son but est de moderniser l'économie. Ses promoteurs - quelques hauts fonction- naires, grands patrons et universitaires se sont appuyés sur le «modèle» des Business Schools nord-américaines alors en cours de réforme. Ils ont utilisé des outils législatifs pour créer des dispositifs comme la FNEGE ou le Centre expérimental de Dauphine. Cette politique ne s'est pas imposée de façon unilatérale : en effet, ce domaine de formation en gestion ćtait déjà «[...] les sciences de gestion constituent aujourd'hui une discipline dominante sur le plan quantitatif mais toujours peu visible sur le plan scientifique »

faut négocier avec les économistes et, en dehors, il faut négocier avec les acteurs consulaires) et une opposition «à l'université au service de l'industrie " acquiert plus d'écho. En outre, cette politique ne s'est pas instaurée de façon linéaire : paradoxalement, c'est la contestation généralisée de 1968-69 qui a permis le développement de la gestion dans les universités françaises.

Longtemps marginales dans les universités françaises, les sciences de gestion constituent aujourd'hui une discipline dominante sur le plan quantitatif mais toujours peu visible sur le plan scientifique. Les transformations universitaires actuelles promues au niveau international (Vinokur, 2001 ; Milot, 2003) les formations considérées comme un service marchand comme un autre, le double mouvement de régionalisation et d'internationalisation des universités, le brouillage des frontières entre formation initiale et continue, etc. - contribuent à modifier les rapports de force au profit de ces disciplines à visée professionnelle. 


\section{Bibliographie}

Bernard M.-Y. (1970), Les Instituts universitaires de technologie, Paris, Dunod.

Boltanski L. (1980), « L'université, les entreprises et la multiplication des salariés bourgeois, 1960-1975 ", Actes de la recherche en sciences sociales, $\mathrm{n}^{\circ} 34$, pp. 17-44.

Bourdieu P. (1989), La noblesse d'État. Grandes écoles et esprit de corps, Paris, Minuit.

Chessel M.-E., Pavis F. (2001), Le technocrate, le patron et le professeur. Une histoire de l'enseignement supérieur de gestion, Paris, Belin.

Cochoy F. (1999), Une histoire du marketing, Discipliner l'économie de marché, Paris, La Découverte.

De Saint-Martin M., Dinu Gheorghiu M. (eds.) (1997), Les écoles de gestion et la formation des élites, Actes du colloque, Maison Suger, MSH, CSEC.

Engwall L., Gunnarsson E. (eds.) (1994), Management studies in an academic context, Stockholm, Almquist \& Wiksell International.

Flouzat D. (1963), L'étudiant économiste. Études, carrières, documentation, 1962-1963, Paris, Cujas.

Fondation nationale pour l'enseignement de la gestion des entreprises (2000), Observatoire des formations à la gestion, document ronéotypé.

Gemelli G. (ed.) (1998), The Ford Foundation and Europe (1950's-1970's). Cross-fertilization of learning in social science and management, Bruxelles, European Interuniversity Press.

Grégoire R. (1964), Les sciences sociales dans l'enseignement supérieur. Administration des entreprises, UNESCO, Paris.

Halsey A. H., Karabel J. (1977), Power and ideology in education, New York, OUP.
Jaccard P. (1962), Sociologie de l'éducation, Paris, Payot.

Kipping M., Nioche J.-P. (1997), " Politique de productivité et formations à la gestion en France (1945-1960) : un essai non transformé », Entreprises et Histoire, $\mathrm{n}^{\circ} 14-15$, pp. 65-87.

Lazuech G. (1999), L'exception française. Le modèle des grandes écoles à l'épreuve de la mondialisation, Rennes, Presses Universitaires de Rennes.

Marco L. (2001) " Le "cocotier" des économistes français avant la réforme de 1968. Eléments pour l'histoire des $5^{\mathrm{e}}$ et $6^{\mathrm{e}}$ sections du CNU ", Les Cahiers de recherche du GRIMM. Groupe de recherche interdisciplinaire en marketing et management, $\mathrm{n}^{\circ} 2$.

Milot P. (2003), « La reconfiguration des universités selon l'OCDE. Économie du savoir et politique de l'innovation ", Actes de la recherche en sciences sociales, $\mathrm{n}^{\circ} 148$, juin, pp. 68-73.

Minot J. (1983), Quinze ans d'histoire des institutions universitaires (mai 1968-mai 1983), Paris, SFA.

Nouschi M. (1988), HEC. Histoire et pouvoir d'une grande École, Paris, Laffont.

Paicheler G. (1992), L'invention de la psychologie moderne, Paris, L'Harmattan.

Papadopoulos G. S. (1994), L'OCDE face à l'éducation 1960-1990, Paris, OCDE.

Passeron J.-C. (1986), « 1950-1980. L'université mise à la question : changement de décor ou changement de cap ? ", in Verger J. (dir.), Histoire des universités en France, Toulouse, Privat, pp. 367-419.

Pavis F. (2003), Sociologie d'une discipline hétéronome. Le monde des formations en gestion entre universités et entreprises en France. Années 1960-90, thèse de doctorat de sociologie Paris I (dir. M. Offerlé). 
Platt J. (dir.) (1963), Problèmes et perspectives de la formation à la gestion des entreprises en Europe, Paris, OCDE.

Prost A. (1997), Éducation, société et politiques. Une histoire de l'enseignement de 1945 à nos jours, Paris, Le Seuil.

Soulié C. (1998), « Le destin d'une institution d'avant-garde : histoire du département de philosophie de Paris VIII », Histoire de l'éducation, ${ }^{\circ} 77$, pp. 47-69.
Tabatoni P. (1996), «Pierre Tabatoni et l'introduction de la gestion en France ", Gérer et Comprendre, $n^{\circ} 44$, pp. 71-84.

Vinokur A. (2002), «Enseignement supérieur : un « changement sans réforme »? », Formation Emploi, $\mathrm{n}^{\circ} 79$, pp. 19-30.

Whitley R., Thomas A., Marceau J. (1981), Masters of business. The making of a new elite? Londres, Tavistock Publications.

\section{Résumé}

\section{L'institutionnalisation universitaire de l'enseignement de gestion en France (1965-1975)}

par Fabienne Pavis

À la fin des années 1960, en France, le développement de l'enseignement universitaire de gestion a bénéficié d'une politique internationale favorable, de la conjoncture de changement de l'université française et de l'engagement de quelques hauts fonctionnaires, grands patrons et universitaires prémobilisés pour cet enseignement. D'autres ont lutté contre l'essor de ces nouvelles disciplines visant un marché du travail non académique. Aujourd'hui, les sciences de gestion constituent une discipline dominante sur le plan quantitatif mais toujours peu visible sur le plan scientifique. 